\title{
Usability and Accessibility of eBay by Screen Reader
}

\author{
Maria Claudia Buzzi ${ }^{1}$, Marina Buzzi ${ }^{1}$, Barbara Leporini ${ }^{2}$, and Fahim Akhter ${ }^{3}$ \\ ${ }^{1}$ CNR-IIT, Pisa, Italy \\ ${ }^{2}$ CNR-ISTI, Pisa, Italy \\ ${ }^{3}$ Zayed University, Dubai, U.A.E \\ \{Claudia, Marina\}.Buzzi@iit.cnr.it, \\ Barbara.Leporini@isti.cnr.it, Fahim.Akhter@zu.ac.ae
}

\begin{abstract}
The evolution of Information and Communication Technology and the rapid growth of the Internet have fuelled a great diffusion of eCommerce websites. Usually these sites have complex layouts crowded with active elements, and thus are difficult to navigate via screen reader. Interactive environments should be properly designed and delivered to everyone, including the blind, who usually use screen readers to interact with their computers. In this paper we investigate the interaction of blind users with eBay, a popular eCommerce website, and discuss how using the W3C Accessible Rich Internet Applications (WAI-ARIA) suite could improve the user experience when navigating via screen reader.
\end{abstract}

Keywords: eCommerce, accessibility, usability, blind, ARIA.

\section{Introduction}

Ever since the late 1990s, a burgeoning number of electronic commerce (eCommerce) websites have attracted countless visitors and consumers.

Usability is a key factor in the success of these eCommerce systems. The term "usability" is precisely defined by the International Organization for Standardization in the ISO 9241, a multi-part standard covering a number of aspects for people working with computers: "the extent to which a product or website can be used by specified users to achieve specified goals with effectiveness, efficiency and satisfaction in a specified context of use" [9]. Many factors can impact user experience, such as rapidity of finding the desired information, efficiency and security when carrying out the transaction, reliability of the delivery service behind the website, etc.

The Web is an important new information resource for people with special needs, since they can retrieve various kinds of information by themselves through the Web.

Blind people can easily access the Web using a screen reader (a software application that interprets and announces what is being displayed on the screen) or non-visual browsers such as voice browsers (the latter possibility does not eliminate the use of screen readers for interacting with the OS and other applications). 
However, the Web is becoming more difficult for blind users as more visual content, such as image links, is being used in Web sites. Blind users' problems can range from mere annoyance at wasted time and effort, to having to abandon a task, or ask for sighted help.

Special-needs persons are a rapidly growing segment of consumers in Europe, mainly due to the increasing median age of the population to be considered when designing eCommerce services.

According to the American Foundation for the Blind, more than twenty million Americans, age 40 and older, are experiencing significant vision loss [1]. And when they attempt to acquire information in the cyber-world, they must overcome a number of barriers that cannot be overcome through training the blind, but only through the intentional development of accessible pages.

In particular, blind people who have mobility problems may successfully utilize on-line eCommerce services. Unfortunately these websites have complex layouts, crowded with active elements that are often difficult to navigate via screen reader. Indeed, it has been acknowledged that blind people face the serious problem that reading certain Web pages is quite difficult $[7,14,17,18]$.

The challenge to web designers is to create a website that is not only visually attractive and informative, but is also accessible and friendly to visually-impaired people.

When designing for blind users, it is necessary to consider the three main interacting subsystems of the Human Processor Model: the perceptual, motor and cognitive systems [4].

Sightless persons perceive page content aurally and navigate via keyboard. This makes the "reading process" time-consuming and sometimes difficult and frustrating, if the contents are not designed with special attention to their needs. Thus, various obstacles make it too hard for blind people to fully understand the structure of a Web page. However, if the Web page is well structured with the help of headings or intrapage links, such as a "skip to main content" link, blind users can easily understand the table of contents or arrive at the main content quickly [14].

The cognition aspect of the interaction is important, since many learning techniques are only relevant to people with good vision and may not apply to someone with a visual impairment. Thus, alternative ways to deliver the identical content should be provided. Furthermore, a blind person may develop a different mental model of both the interaction and the learning processes, so it is crucial to provide an easy overview of the system and contents.

Non-visual perception can lead to:

\section{Content serialization.}

A screen reader reads the contents sequentially, as they appear in the HTML code. This process is time-consuming and annoying when part of the interface (such as the menu and navigation bar) is repeated in every page. As a consequence, blind users often stop the screen reading at the beginning, and prefer to navigate by Tab Keys, from link to link, or explore the content row by row, via arrow keys.

2. Content and structure mixing.

The screen reader announces the most important interface elements such as links, images, and window objects as they appear in the code. For the blind user, these elements are important for figuring out the page structure, but require additional cognitive effort to be interpreted. 
3. Table.

If the table's content is organized in columns, the screen reader (which reads by rows) announces the page contents out of order; consequently the information might be confusing or misleading for the user.

4. Lack of context.

When navigating by screen reader the user can access only small portions of text and may lose the overall context of the page; thus it may be necessary to reiterate the reading process.

5. Lack of interface overview.

Blind persons do not perceive the overall structure of the interface, so they can navigate for a long time without finding the most relevant contents.

6. Difficulty understanding UI elements.

Links, content, and button labels should be context-independent and selfexplanatory.

7. Difficulty working with form control elements.

For example, the new JAWS (an acronym for Job Access With Speech, a popular screen reader) version (v. 10) simplifies the interaction with forms since it can automatically activate the editing modality (for text input) when the virtual focus arrives at the text box (for instance when the user presses the Tab key). However, with previous screen reader versions the user may experience great difficulty, since switching between exploration and editing modalities is required (i.e. form mode on/off).

8. A blind person is unable to access multimedia content such as video streaming, video conferencing, and captioning.

If an alternative description is not present, the user may lose important content.

In this paper we analyze the accessibility and usability of eBay, a popular eCommerce website. Specifically, we show the interaction with the home and result pages. Then we discuss how WAI-ARIA [19], the suite developed by the Web Accessibility Initiative (WAI) group of W3C, might facilitate interaction for the blind. Section 2 presents related works, Section 3 describes the exploration of eBay via screen reader, highlighting potential problems and Section 4 introduces the WAIARIA suite, discussing the advantages of structuring the content in landmarks/regions.

\section{Related Works}

It is essential to implement both usability and accessibility principles when designing a user interface (UI). Accessibility is a basic pre-requisite for allowing users to have access to the web page content, while usability provides online users with simple, efficient, rapid and satisfying navigation and interaction.

Navigation is vital for special-needs persons, and especially for the blind, since it is crucial for them to be aware of their current location on the webpage and how to return to the beginning, or how to reach a certain point in the material [5].

ECommerce systems pose new challenges with respect to classic user-centered product design, where the target is a set of homogeneous users. 
Various studies investigate the usability of eCommerce systems and include a general discussion on accessibility, but to our knowledge only a few focus on totally blind persons $[16,2,15,8]$.

Petrie et al. [16] presented the results of accessibility testing of 100 websites with users with various (visual, motor and perceptual) disabilities, showing that websites that are accessible for differently-abled users can also be visually pleasing.

Concurrently, they addressed different aspects of accessibility that do affect visual design, such as visual structure, color contrast, and text size, aspects of design that can affect all users, not only the disabled. Specifically the study tested 100 websites spread out over five sectors (including eCommerce) with automated verification and user testing involving 51 differently-abled users, including 10 totally blind users.

Of the eCommerce websites analyzed, eBay.co.uk was chosen as a case study. Although pages were cluttered, authors registered a $100 \%$ task success rate among users, including the totally blind, observing that a complex layout is possible without necessarily compromising accessibility. However, since details of the user test are not specified it is difficult to evaluate the potential difficulties of each task: where the target is located in the site, the number of steps needed to complete the task, ease of use and performance. This affects user satisfaction. Concerning this last point the authors recorded that the blind encountered more difficulties than did other differently-abled users.

Organizing a page in logical sections enhances the experience of the blind user when navigating a page in two ways: it provides a page overview and offers the possibility of jumping from section to section. Specifically, heading levels may improve navigation since screen readers have special commands for moving from one heading to another.

Brudvik et al. [2] present an interesting study on how sighted users associate headings with a web page, observing very different results depending on factors such as whether the page has a hierarchic structure, how users identify sections, etc. Furthermore authors applied techniques of information retrieval (i.e. training data and a classifier), developing a system for automatically inferring from the context (font, size, color, surrounding text, etc.) if a phrase "works semantically" (and may function) as a heading, and dynamically adds the heading level using Javascript. The system called HeadingHunter was evaluated using human-labeled headings gathered from the study and showed high precision (0.92 with 1 the max).

Most common website usability factors involve meeting business objectives while providing a satisfying user experience. Therefore, accessibility should be seen as a challenge to designers and implementers rather than as a constraint.

Parmanto et al. showed that simplification and summarization may enhance usability of crowded web sites for the visually impaired [15]. To simplify user interaction via screen reader, irrelevant content is removed according a strategy that also takes into account the genre of the website (eCommerce, News, etc.). Furthermore, summarization provides users with a page overview that facilitates user orientation.

A pilot usability study conducted by authors with two visually impaired subjects on the original and the transformed Yahoo!News website, offered promising results.

In general, different approaches may be applied to transform on-fly web pages, in order to improve interface usability and content accessibility, such as using an intermediary proxy server or specific client-side solutions (e.g. modified browser, 
plug-in, etc.). However, the automatic simplification or on-fly transformation, applied to any website, might lead to the accidental removal of content interesting for the user.

We suggest the use of WAI-ARIA to reach a similar result (page overview, easy orientation, access to main contents, etc.) while maintaining the website content unaltered.

Last, an interesting study by Gladstone et al., investigates the use of a natural language interface for simplifying interaction for the blind [8]. Specifically, the Online Shop of the Royal National Institute of Blind People was redesigned, creating fully accessible and usable interfaces while guaranteeing that they were visually attractive. The use of natural language in association with a user profile allows the system to respond to a greater range of phrases, improving its effectiveness and efficiency.

\section{Exploring eBay via Screen Reader}

For this study, eBay.com was selected to evaluate accessibility and usability of eCommerce websites via screen reader. We chose eBay.com as a case study due to the simplicity and clarity of its graphical user interface and because it is one of the most reputable websites in the online auction business. The richness of a UI is the strength of an eCommerce service, but the environment's complexity can create difficulties when interacting via screen reader.

\subsection{Evaluation Methodology}

The eBay home and result pages were analyzed with a usability inspection, carried out independently by three of this paper's authors, who are also accessibility experts.

The pages were navigated using the screen reader JAWS for Windows (http://www.freedomscientific.com) v. 9.0 and 10. We used both the MS IE version 7.0 and the Mozilla Firefox version 3.0.5 browsers.

The test was carried out by the authors independently and observations were annotated by everyone. Afterwards, outcomes were compared, discussed and integrated in a face-to-face meeting.

One author has been totally blind since childhood and uses the JAWS screen reader every day; thus she knows this tool's functions very well and is able to use advanced commands. By analyzing the test results we noticed that in spite of her great expertise using JAWS, she was unable to perceive the exact structure of the layout.

The sighted authors carried out the same test mainly using JAWS basic commands (carried out with PC screen turned off).

Therefore, integrating both these outcomes led to a more accurate analysis. The different experiences of the authors when using JAWS allowed us to cover a variety of interaction modalities: i.e. basic commands, simulating the level of novice users, and advanced screen reader functions for experienced users.

\subsection{Interacting with the eBay Homepage}

In order to evaluate eBay navigation aspect, we accessed the content of the home page to monitor the accessibility and usability aspects via the JAWS screen reader. In our evaluation, we referred to the usability criteria reported in [13], [14]. 


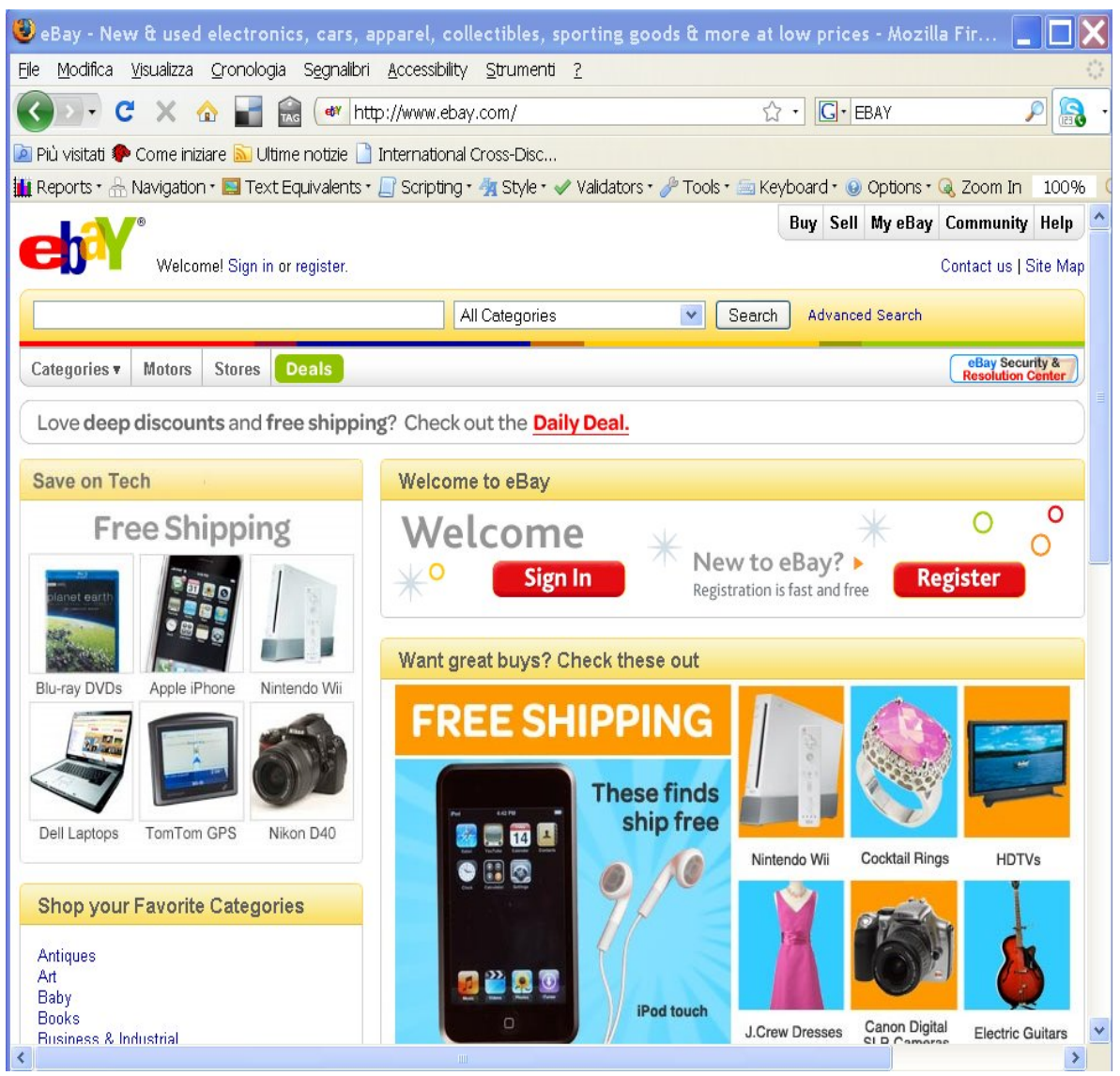

Fig. 1. eBay home page screenshot

By accessing the eBay home page (Fig. 1), we observed the following features:

- Number of links

Over 100 links are detected by JAWS when loading the Web page. There are far too many links to be navigated, and it is hard for a blind user to orientate him/herself among the links or find the interesting ones (Fig. 2). The exploration could be simplified by reducing the number of links, or by grouping them according to the interest.

- Headings

No heading level is detected by JAWS. Headings can help blind users understand the main structure of the page content. Headings could give a blind user an overview of the content by executing a specific screen reader command to list all headings within the page - i.e. all main areas in which the content can be structured. For instance, that effect can be obtained with the JAWS screen reader by pressing Insert+F6. Moreover, a more appropriate effect can be provided via ARIA landmarks [19] (see Section 4 for details). For instance, in [3] the authors 


\section{Links List}

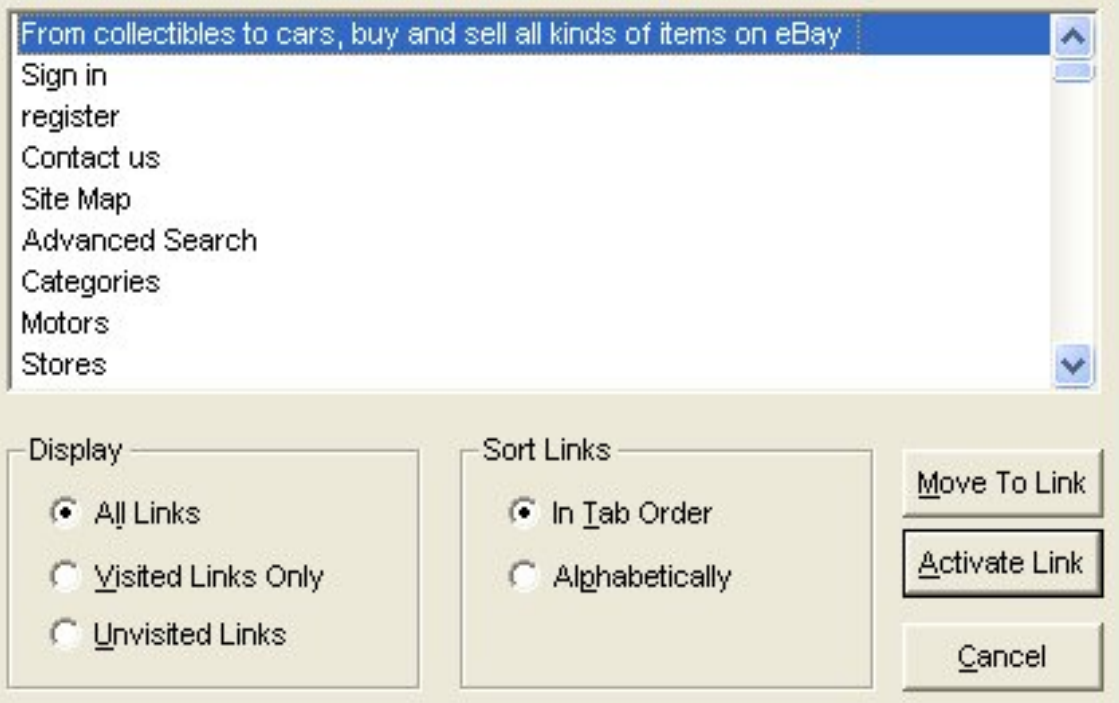

http:Mwww ebay.com

Fig. 2. eBay home page links list detected by JAWS

used landmarks for partitioning the page content using Wikipedia Editing Page as a case study.

- Search functions

By navigating content in a sequential way or by using advanced interacting JAWS commands -- e.g., letter "e" to move onto edit fields, or "fo" to navigate among the form elements -- the blind user encounters an edit field. By exploring around that edit field, he/she can guess that the field is used to search for something within categories; in fact, below the edit box there are a combobox for categories and a "search" button. A specific label for the edit field should be added to make the UI clearer.

- Graphical map

The page contains some links recognized as a map, in which alternative descriptions are not provided. After the sentence "Welcome to eBay", the JAWS screen reader detects three graphic map links: image map link "ws/eBayISAPI.dll?SignIn\&ru=http $\% 3 \mathrm{~A} \% 2 \mathrm{~F} \% 2 \mathrm{Fwww}$.ebay" (corresponding to the "Sign In" Button), image map link "ws/eBayISAPI.dll?RegisterEnterInfo" (corresponding to the "Register" Button) and Image map link "246". For a blind user those links are useless. Fortunately these links are also duplicated close to the logo (upper left side), thus the screen reader is able to intercept and communicate them to the user. 
- Navigation links and shortcuts

Neither local navigation links (e.g. "skip to content") nor shortcuts to activate main links are used in the home page. The user has to read the page in a sequential way by using the arrow keys or the Tab key, wasting time.

\subsection{Exploring a Search Result}

Let us now consider the result page when looking for a specific product. Suppose one is interested in buying audio books. A blind user accesses the home page, moves onto the Edit field, and writes the word "audiobooks". After the user presses the Enter key, the result page is loaded. In this case a heading associated with the desired word is used. Thus, as soon as the page is loaded, by typing the letter " $h$ " the JAWS virtual focus moves towards the result main section. From this point, the user can explore the items found in a sequential way.

Fig. 3 shows the result page; the search query is "audiobooks". Fig. 4 reports a segment of page content read by JAWS.

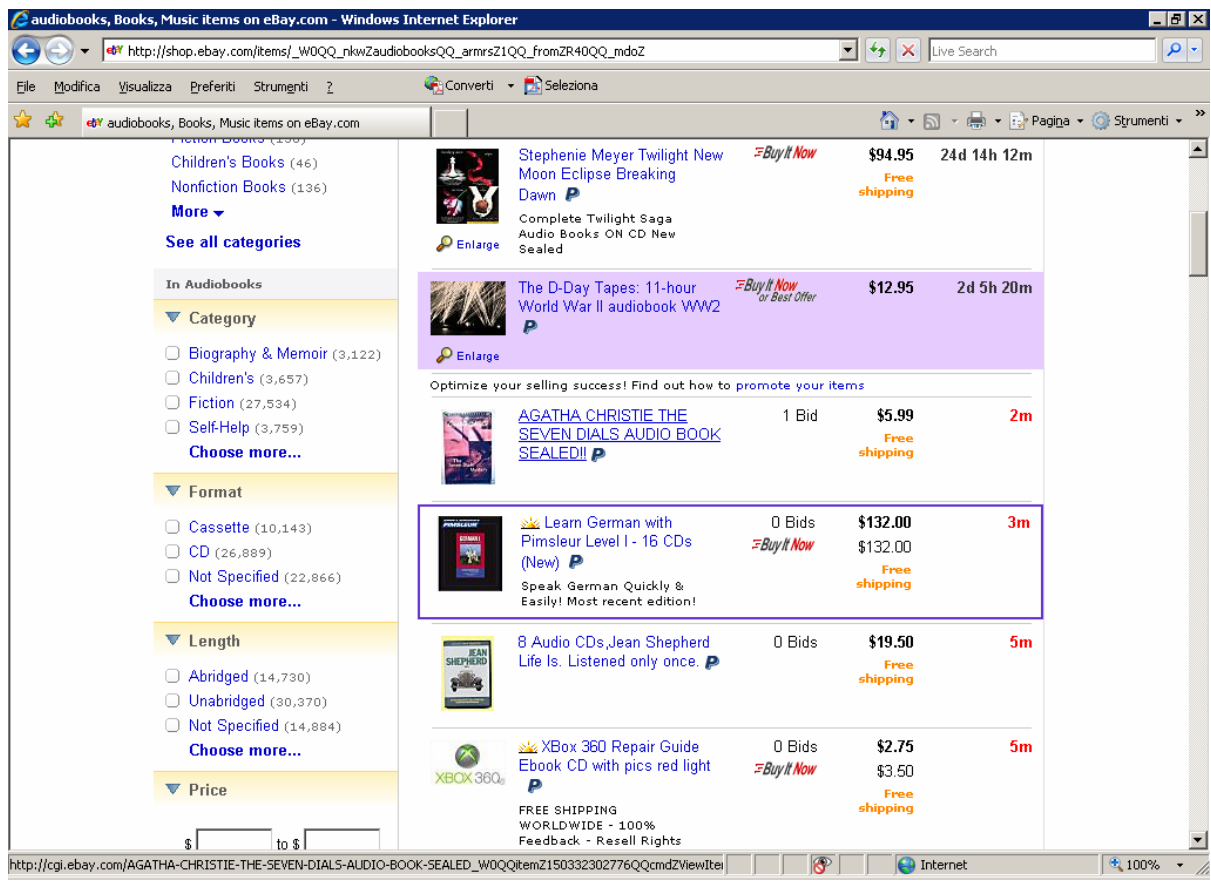

Fig. 3. eBay result page screenshot obtained with the search string "audiobooks"

The main features observed for the result page can summarized as follows:

- No rendering structure

All the found items or a subset of them are rendered in a sequential way without any structure (e.g. an item list, a heading for each product, and so on). Indeed, the default rendering customization is settled on "List", but the list is not implemented 


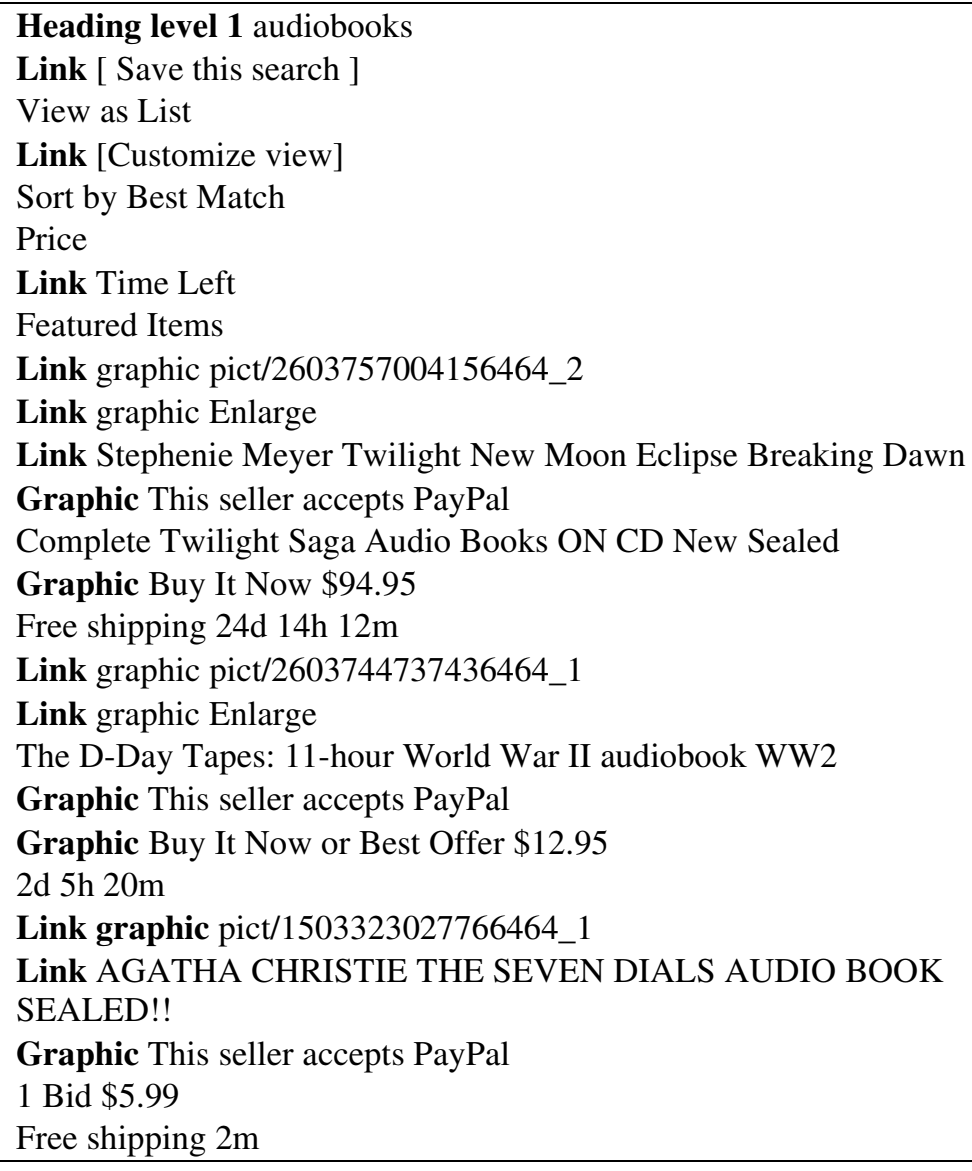

Fig. 4. Segment of the result items read by the JAWS screen reader

in a standard way (i.e. with the <ul $>$ HTML tag). In other words, the user must read all elements by the arrow keys, in order to explore their characteristics.

- Graphical functional links

Each result element has a graphical link with no alternative descriptions, which should be used to access a specific product.

- Result pages

Links pointing to the result pages are not clearly grouped and shown in a way that allows them to be reached quickly.

\section{Discussion}

Using ARIA would enhance eBay's usability in many ways, as explained and exhaustively illustrated by the ARIA best practices document [19].

First of all, structuring the web page in logical sections can provide blind users with a page overview. An important guideline for simplifying user navigation is 
related to the logical partitioning of content. As mentioned in the previous section, no heading has been used to partition the page into different areas.

In this perspective, landmarks could guide the user to the main areas in which the page can be split. In particular, the eBay home page could be partitioned into areas such as "Login", "Search", "Actions" (e.g. "buy", "sell”, "my ebay", etc.), "Great buys", "From our Sellers", "Shop favorite categories", and "Choose language". In this way, the user could quickly refer to the desired page section.

Analogously, the result page could be split into logical sections.

ARIA offers the following default landmarks: application, banner, main, navigation, search, complementary, contentinfo, log and status. If the logical section does not fit one of these landmarks, it is possible to use the role region and specify a title:

$<$ div role="main" $>\ldots<$ div role="region" title="Great buys" $>\ldots$

The "flow to" property may be used to force the screen reader to navigate these sections in an order (established by the web designer) that should reflect how the user navigates the page (e.g. <flow to="Great buys").

Having common standard navigation landmarks with the same meaning would provide a blind user with a consistent navigation experience on every website. Furthermore, cognitive- and learning- disabled users could take advantage of collapsed/expanded regions to manage the amount of information processed at any one time [19].

These basic considerations for improving interaction via screen reader should be integrated according to the observations reported below.

Making eCommerce websites suitable for the abilities and skill levels of all users is a great challenge. Obviously, the needs of sighted users as well as the blind must still be considered when defining the graphical interface.

Information should be provided through both visual and auditory channels, the design should be optimized for reading via screen reader, the interface must be easy to use via keyboard, and no additional cognitive effort should be required of the blind user.

In this study we investigated the interaction of blind users with eBay, via screen reader. We analyzed the eBay home page and one result pages to verify the usability.

The results have shown that both pages contain some features that could be improved. Specifically, we suggested ways in which ARIA would enhance usability in these two important pages.

In conclusion, we believe that our suggestions could have general applications, and that applying ARIA would enhance usability via screen reader in any eCommerce environment.

\section{References}

1. American Foundation for the Blind, http://www.afb.org/Section.asp? SectionID=15

2. Brudvik, J.T., Bigham, J.P., Cavander, A.C., Ladner, R.E.: Hunting for headings: sighted labeling vs. automatic classification of headings. In: 10th international ACM SIGACCESS conference on Computers and accessibility, pp. 201-208 (2008)

3. Buzzi, M., Buzzi, M.C., Leporini, B., Senette, C.: Improving Interaction via Screen Reader Using ARIA: An Example. In: 18th International World Wide Web Conference (WWW 2009) Developers Track (2009), http://www2009.org/pdf/www09dev _proceedings.pdf 
4. Card, S.K., Moran, A., Newell, T.P.: The Psychology of Human-Computer Interaction. Lawrence Erlbaum Associates Inc., New Jersey (1983)

5. Debevc, M., Verlic, M., Kosec, P., Stjepanovic, Z.: How Can HCI Factors Improve Accessibility of m-Learning for Persons with Special Needs? In: Stephanidis, C. (ed.) HCI 2007. LNCS, vol. 4556, pp. 539-548. Springer, Heidelberg (2007)

6. Disability Rights Commission: The web access and inclusion for disabled people. Technical report, Disability Rights Commission (DRC), UK (2004)

7. Fukuda, K., Saito, S., Takagi, H., Asakawa, C.: Proposing new metrics to evaluate web usability for the blind. In: CHI 2005 on Human factors in computing systems, pp. 13871390. ACM, New York (2005)

8. Gladstone, K., Rundle, C., Alexander, T.: Accessibility and Usability of eCommerce Systems. In: Miesenberger, K., Klaus, J., Zagler, W.L. (eds.) ICCHP 2002. LNCS, vol. 2398, pp. 11-18. Springer, Heidelberg (2002)

9. International Standard Organization (ISO), ISO 9241-11: Ergonomic Requirements for Office Work with Visual Display Terminals (VDTs), Part 11: Guidance on Usability, 1st ed., International Organization for Standardization, Geneva, CH, 1998-03-15 (1998)

10. Harrison, C., Petrie, H.: Severity of usability and accessibility problems in eCommerce and eGovernment websites. In: People and Computers XX. Engage, pp. 255-262. Springer, Heidelberg (2005)

11. Huang, A.W., Sundaresan, N.: A semantic transcoding system to adapt Web services for users with disabilities. In: Fourth international ACM conference on Assistive technologies, pp. 156-163 (2000)

12. International Standards Organization (1992 - 2000). Standard 9241: Ergonomic requirements for office work with visual display terminals, http: / www. iso.org

13. Leporini, B., Andronico, P., Buzzi, M., Castello, C.: Evaluating a modified Google user interface via screen reader. Universal Access in the Information Society 7(1-2) (2008)

14. Leporini, B., Paternò, F.: Applying web usability criteria for vision-impaired users: does it really improve task performance? International Journal of Human-Computer Interaction (IJHCI) 24(1), 17-47 (2008)

15. Parmanto, B., Ferrydiansyah, R., Saptono, A., Song, L.: AcceSS: accessibility through simplification \& summarization. In: 2005 International Cross-Disciplinary Workshop on Web Accessibility (W4A), pp. 18-25. ACM Press, New York (2005)

16. Petrie, H., Hamilton, F., King, N.: Tension, what tension?: Website accessibility and visual design. In: 2004 international cross-disciplinary workshop on Web accessibility (W4A), pp. 13-18 (2004)

17. Takagi, H., Asakawa, C., Fukuda, K., Maeda, J.: Accessibility designer: visualizing usability for the blind. In: 6th international ACM SIGACCESS conference on Computers and accessibility, pp. 177-184 (2004)

18. Theofanos, M.F., Redish, G.: Bridging the gap: between accessibility and usability. Interaction 10(6), 36-51 (2003)

19. W3C. WAI-ARIA Best Practices. W3C Working Draft (February 4, 2008), http: / / www.w3 .org/TR/wai-aria-practices/ 